\title{
Index of people Part II
}

The index covers the main text, and excludes front matter, bibliographic references and bibliography, as well as names in the titles of works and illustration credits. People are referenced by their last and first name(s). For Zulu names we follow Laband $(1995,505)$ and reference them 'under the stem and not under the prefix'. More search options are provided by the open access book.

Ackerman (née Potgieter), Katharina Fredrika (D.P.) 53, 54, 55, 73, 94

Ahlers, H. 422, 469

Ahlers Breedt, H. 154

Albagh (Albach), Lourens Marthinus (Louw) 72, 73

Amshewitz, John Henry 119-121

Anderson, Rufus $131 \mathrm{n} 237$

Archbell, James 131 n 237, 141, 145, 147, 149-152, 484

Atkins, Harry 498 notes 1157 and 1158, 499

Attalus I 95

Augustus, Caesar 426

Baines, Thomas 169, 170, 199 n 373, 309-311

Bantjes, Jan Gerritse 222 n 434, 224, 232, 248, 252 n 559, 260 n 586, 264, 282 n 634, 283, 411 n 879, 426, 427, 428, 430, 432, 433, 447-452, 456, 473, 482

Barends, Barend 575

Barnard, Chris see Van der Walt, Gert

Basson, Martin Johannes Adriaan 43 n 71; 79, 103, 141, 205, 269, 291 n 656, 295, 296, 303, 415, 437, 463, 467, 496 n 1153, 507, 545, 561

Basson, N.J.S. (Nicolas Johannes Salomon?) 592

Becker, Peter 217, 289 n 654

Behrens, Carl 476

Bell, Charles Davidson 91, 92, 132

Berning, Gillian 265 n 600

Bezuidenhout, Cornelia Sophia 320

Bezuidenhout, Daniel Pieter 166 n 320, 167 n 325, 220 n 424, 225, 265, 319, 342, 357 n 801, 358 n $804,538,556$

Bezuidenhout (née Smit), Elizabetta Cecilia 320 Bezuidenhout (née Liebenberg), Elizabetta Johanna 320 Bezuidenhout, Hendrik Cornelius 320

Bezuidenhout, Maria Adriana 320

Bezuidenhout, Petrus Johannes 320, 359 n 806

Bezuidenhout, Rachel Jacoba 320

Bezuidenhout, Wynand Frederik 330

Biggar, Alexander Harvey 221, 447

Bird (née Williams), Jane 225, 279-282, 319 n 740

Bird, John 222, 248, 251, 426, 478 n 1046, 490

Bloem, Jan 575

Blommaert, Willem 231

Booysen, C.M. 473, 474

Boshof(f), Jacobus Nicolaas 43 n 70, 221 n 425, 320, 326, 341 n 773, 375, 377, 480, 519, 521

Boshof, Johan Christoffel 489, 490

Bosman, J.J. 422

Bosman, Nollie 162

Botes, F.J. 592

Botha, Elizabeth Helena 92
Botha, Elizabetta Johanna 320

Botha, Hendrina $58 \mathrm{n} 95$

Botha, Lea (Leah) 177, 178

Botha, Louis 299

Botha, Michiel Coenraad 300

Botha, Pieter Johannes Hendrik 51, 57, 73

Botha (née Bezuidenhout), Susanna Margarita 320

Botha, Zabeth 246 n 520

Boyce, William 215 n 395, 249 n 536, 321 n 754

Brand, Christoffel Joseph 223, 244, 299, 300, 482

Brand, Johannes 299

Brand, P.A. 223, 244, 250

Brayhurst, Vicar 493

Breda, Michael sr 534

Breedt (student) 154, 195

Breytenbach, Izak Johannes 320, 326

Broers, Sannie 325

Bronkhorst, Johannes Gerhardus Stephanus 90, 147, 148

Brookes, Edgar Harry 329

Brownlee, James 281

Brownlee, John 221 n 427

Buchanan, David Dale 490

Buchanan, William Herbert 493 notes 1134 and 1136

Bull, John 593

Burger, Jacobus Johannes 232, 246, 247, 248, 475 n 1032, 481, 484

Burger, Schalk Willem 360 n 809, 393, 409

Burgers, Thomas François 75

Burton, James Edward 35

Buthelezi, Mangosuthu Gatsha 457

Cachet, Frans Lion 433 n 939, 489, 588

Cane, John 282

Cannon, Richard 595 n 1372

Carey, Margaret 273 n 608

Celliers, Charl see Cilliers, Sarel

Cetshwayo 265 n 600

Chaka see Shaka kaSenzangakhona

Champion, George 221

Charters, Samuel 226-229, 231, 233-235, 244-245, 250, 393, 447 n 955, 448 n 967

Chase, John James Centlivres 147 n 272, 166 n 322, 199, 219 n 420, 222

Churchill, Marianne 485-486, 488

Cilliers, Sarel Arnoldus 89-99, 135 n 254, 147, 148, 150, 224, 282 n 634, 376 n 837, 377 n 841, 393, 409, 415, 417-433, 447, 448, 473, 482, 484, 489

Clarkson, Charles Francis 329

Cloete, Henry 250, 251, 252 n 556, 283, 394, 395, 397, 476-480, 483 n 1076, 484, 491, 554 n 1275, 574 
Cloete, Josias 573,574

Coertzen, Pieter 294 n 677

Coetzee (née Botha), Anna S. 148

Coetzee, Jan 109

Coetzee, Marius 422

Coetzee, Nicolaas J. (Nico) 453

Coetzer, Philippus Jeremias 375, 376, 456 n 999, 529 n 1204, 537 n 1235

Coetzer, Willem Hermanus $3,4,9,11,12,13,15,17,27$, $30,31,33,36,47,50,51,57,58$ n 96, 61, 64, 65, $79,82-85,98,103,106,107,115-118,127$ n 235, $129,141,144,145,151,157,160,161,163,168 \mathrm{n}$ $336,177,189,192,193,195,200,205,208,209$, $211,222,264,265,269,272,273,275,281$ n 627 , 283, 285, 286, 296, 300, 303, 308, 309, 310, 311, $313,315,326,330,339,347,350,351,352,369$, $383,386,387,389,395,401,404,405,407,415$, 418, 419, 421, 437, 440, 441, 442, 452, 463, 466, 467, 469, 511, 517, 518, 519 n 1196, 525, 528, 529, 530, 538, 539, 540, 541 n 1253, 549, 561, 564, 565, 566, 577, 581, 584, 585, 587, 593

Combrinck, J (Oom Commie) 359

Combrink, H.J. 442

Cortez (Hernán Cortés de Monroy y Pizarro Altamirano) 153

Cory, George Edward 37, 38, 73 n 115, 221, 231, 232-233, 250, 252, 253, 256, 260, 261 n 587, 266 , 282 n 630, 283 n 635

Cronin, Michael (Mike) 288, 289 n 653

Cronje, Pierre 501

Crouch, Neil $11 \mathrm{n} \mathrm{3,563}$

Cubbin, Anthony E. 261

mDada see Manzi Mdada

Da Fonseca, João de Barros Ferreira da Fonseca 73 Da Fonseca (Fon-secca), Maria Eunia 73

Da Silva Pereira, Manuel 73

David (Old Testament) 119

De Beer family 199, 342

De Jager, Christoffel (Stoffel) 569

De Jager, Jan 360

De Jongh, P.S. 360 n 807

De Klerk, Lourens Christian 341, 342, 343

De Kock, Jos. (Josephus?) 425

De Lange, Johan Hendrik (Hans Dons) 409, 449

Delegorgue, Adulphe 81 n 127, 91 n 159, 165 n 316, 199, 201 n 377, 222 n 434, 233, 234, 393 n 853, 449 n

977, 451 notes 984 and $986,476,529,534-537$, 540,573

De Villiers, Natalie 94

De Wet, Magdalena Johanna see Retief, Magdalena Johanna

Dingane kaSenzangakhona (Dingaan, Dingarn) 131, 157, 165-168, 173, 185, 199, 205-266, 269, 271, 275, 279-289, 292, 295, 296, 303, 319, 326, 341, $357,360,375,393,395,409,411,415,426,428$, $430,437,447-450,488,495,519,525,527,530$, $533-540,545-557,561,573$

Don Quixote 593

Doucakis, Alkis 131
Drury, Edward Guy Dru 298

Du Plessis, A.J. 43 n 71, 79, 103, 141, 205, 269, 303, 415, 437, 463, 507, 545, 561

Du Plessis, Jan 283, 481

Du Plessis, L. Theodorus 449 n 977

Du Plooy Erlank, Wilhelm Jacobus (poet Eitemal) 94

Du Preez (Miss) 340

Du Preez, Pieter Daniël (Andreas Salomon) 241 n 502, 232, 242, 243, 246, 247, 248, 250

Du Toit, André 426 n 902

D’Urban, Benjamin Alfred 55 n 83, 89, 147

Duvenage, H.J.P. 94

Egersdörfer, Heinrich 79, 83, 84, 87, 91, 132

Ehud 116, 118

Eitemal see Du Plooy Erlank

Eksteen, Louis J. 498 n 1157, 499, 521

Engelbrecht, Stephanus Petrus (Fanie) 320 n 751, 329, 474, 475, 488, 489, 585

Enslin, Johan Adam 589

Ephialtes 360, 361

Esterhuizen, Kobus 457

Etherington, Norman 42, 81 n 127, 87 n 135, 89 n 139, 91 n 159, 219 n 421, 450 n 980, 573, 575 n 1320

Evatt, Francis $221 \mathrm{n} 425$

Eyssen, Steven 109

Faure, A. 478, 481

Faure, David Pieter 299

Faure, Hendrik Emmanuel 485, 490, 491

Faure, P. 481

Faye (Feye, Mr) 211, 277

Ferreira, Marthinus Philippus Cornelis 490, 491

Franken, John Lambert Machiel 298

Fourie, Philippus Jacobus 451

Fuller, Claude 57 note 89 and 90,68-73, 189

Furstenburg, J.P. 592

Gamitto, Antonio Candido Pedroso 63-65, 69, 72, 73, 74

Gardiner, Allen Francis 216, 217, 225 n 450, 275 n 610, 282, 289 n 654

Geer, Karel 342 n 782

Genghis Khan 153

Gerdener, Gustav 92, 93, 429, 431

Ghubeni, N. 263

Gideon 116, 118, 185 n 358

Giliomee, Hermann Buhr 57 n 91, 81 n 127, 89 n 140, 90 n 157, 116, 117, 594

Gledhill, Eily and Jack 113 n 196, 114 n 208, 115, 181, 183 n 357, 185 n 358, 214, 217, 297 n 698, 298 n 702

Glenn, Ian 153 n 304

Glover, Michael 215 n 396

Godide kaNdhleha 554

Godlonton, Robert 113 n 196, 297

Goldman, Lex 253

Gordon, G.H. 487, 488

Gouws (widow) 393

Grey, Henry George 590, 593 
Greyling, Abraham Carel 166, 183, 235, 236, 239, 254, 255, 258, 259

Greyling, Jan Christoffel 167 n 330, 183, 473 n 1019

Greyling, Magdalena Johanna see Retief, Magdalena Johanna

Greyling, Pieter Jacobus (Piet) 473, 502

Grobbelaar, Johannes Hermanus 592

Grobbelaar, J.N. 592

Grobbelaar, Nicolaas Johannes 590, 592

Grobler, Jackie 38, 69 n 109, 279 n 615, 519 n 1197

Grotius, Hugo 116-117

Guldenpfennig, Helene 340

Gutsche, Thelma 35

Hall White, Henry 292

Halstead, Thomas 207, 212, 220, 263, 266, 269, 282, 357

Haswell, Robert F. 475 n 1031, 478 n 1046, 480, 481, 492

Hattersley, Alan Frederick 476, 575 n 1314

Hattingh, Johannes Dewald (Dewalt) 409

Hattingh, Johannes Hendrik 84 n 132, 152, 375, 448 n 964

Havemann, Johan Heinrich Carl 321

Henning, Cosmo Grenville 297

Henning, Elrica 264 n 593, 291 n 658, 292 n 674, 369 notes 817-818, 478 notes 1044-1045, 484 n 1079 , 487, 492 n 1130, 493, 494, 498 notes $1157-1158$

Herod (New Testament) 330

Herold, T. 481

Hertzog, James Barry Munnik 31, 266

Heymans, Riana 342, 343, 395 n 860, 521 n 1201

Hobbs, Philippa 11 n 3, 563 n 1290

Hofmeyr, Jan Hendrik 328, 329

Hofstede, Helperus Johannes 148 n 284, 232, 251, 291-297, 419, 425, 429, 431, 432, 452 n 989, 489

Hogge (née Magniac), Helen Julia (Nina) 590-594

Hogge, William Samuel 590-595

Holloway (Dr) 513

Holloway, Alida 513

Holloway, Tienie 109

Horak, Ena 325

Horak, Johan 325

Howard, Ingrid 595 n 1374

Hulley (Halley), Richard Brangan 217, 221 n 428, 225 , 249 n 536, 251, 280 n 620, 281, 282

Hurter, J.S. 328

Ingram, Forsyth 488

Jacobs, Louis 94, 95

James Stuart (Archive) see Stuart, James

Jana, Aia 321

Jansen, Ernest George 3, 4, 9, 27, 47, 61, 79, 103, 141 , $149,157,161,168,173,185$ n 360, 189, 205, 209, $269,300,303,313,315,328,329,347,365,369$, 383, 394, 395, 401, 415, 425, 429, 430 n 916, 432, 437, 442, 456, 463, 487, 493, 496, 498 n 1158, $499,507,511,515,517,518,521,525,538,549$, 561,581
Jansen, Martha Mabel (Mabel) 498 n 1158, 501

Jeppe (Mr) 232, 246 n 520, 248, 251, 257, 258, 260

Jervis, Henry 230, 475, 476

Jesus 116

Jolly, Pieter 207 n 383

Jooste, Marius 457

Jooste, Martin 54

Joseph (New Testament) 119

Joubert, Anna Margaretha 294

Joubert, Hendrik 98 n 185

Joubert, Marie 569

Joubert, Petrus (Pieter) Jacobus 321, 322 n 756, 427

Joubert, Stephan 162, 177, 178

Joubert, Stephanie 177,178

Joubert, Willem François 589, 592

Joyce, Robert 447

Julianus (Juliavius) 235, 236, 239, 243, 244, 247, 254, 255

Juta, Jan 30, 31, 33, 43, 119, 121, 557, 558

Kaliphi 89, 91

Kammeyer, Gerard 195, 391

Kammeyer (Mrs), I. 195

Kemp, Jan Christoffel Greyling 421, 422

Kestell, Gertruida Anna (Trudie) 407

Kestell, John Daniel 407, 495, 497

Keur, Jacob and Hendrik 39, 42

King, Richard 319 notes 737 and 743

Kirchhoff (née Bose), Anna-Maria 19, 22, 513

Kirchhoff, Peter 9, 14-23, 109, 168 n 336, 195, 329, $359,387,423,437,439$ n 944, 441-445, 452, 453, 463-471, 507-515, 518, 581-587, 594-596

Kirchhoff, Vera 19, 22, 507-513

Kirchhoff, Werner 94, 95, 177 n 342, 357, 359, 469, 513, $530 \mathrm{n} 1206$

Kirkman, Joseph 221

Kleinhäns, Billie 177, 178

Klopper, Henning Johannes 36, 38, 98, 432, 489 n 1106

Klopper, Sandra 207 n 380, 265

Kloppers, J.C. 592

Kriel, Justa (married name Proctor De Villiers) 195

Kriel (Ms, student) 94

Krige, Eileen 127 n 234, 207 n 380

Kruger, Frikkie 19, 22, 111 n 191, 127 n 235, 141-146, 189-201, 205-212, 263, 269-277, 286, 287, $335-340,515$ n 1180, 518, 525-530, 540, 541, 561-570, 587

Kruger (Frikkie Kruger’s father) 569, 587

Kruger, G.J. 592

Kruger, Johannes Paulus (Paul) 75, 81, 87, 94, 95, 98 n 184, 119, 251, 252, 253,489, 585

Kruger, Mietje (Maria?) 393

Laband, John 81 n 127, 131 n 239, 216 n 402, 356, 409 n 872, 447 n 960, 450, 475 n 1029, 480, 481, 492, 533 notes 1210 and $1216,537,552,553$ notes 1262 , 1266 and $1267,556,573$

Labuschagne, J. Andrë 476, 478 n 1046, 482 n 1070, 483, 491 n 1126 
Landman, Debora Jacoba see Retief, Debora

Landman, Karel (Carel) Pieter 185 n 358, 226-228, 232-234, 237, 239, 241-243, 246, 248, 261, 409, 427

Landman, Willem Adolph 185

Lawson, C.C.P. 595, 596

Lea, Henry 370 n 824, 371, 372, 415

Leeu (Gerard Moerdyk's dog) 143, 145, 153, 154

Lehman, J.M. 592

Leyds, Willem Johannes 232, 251-253, 255-257, 589 n 1326

Liebenberg, Barend Jacobus 408, 409, 411 n 879, 427, 430 n 916, 446, 447, 448 n 964, 449, 536 n 1228, 539 n 1246, 575 n 1311

Liebenberg, Barend Johannes sr and family 89,167 (with n 325), 219, 235, 236, 239, 243, 247, 254, $255,258,259,330$

Liebenberg, C. 181

Liebenberg, Elizabetta Johanna see Bezuidenhout, Elizabetta

Liebenberg-Barkhuizen, Estelle 369

Lindeque, Alie 391

Lind(e)que, Lydia 391

Lindley, Daniel 131 n 237, 217-219, 233 n 473, 242 n 503, 260, 323 n 760, 483, 484, 485

Lindley, Martha Jane 485, 487, 488

Lindsey, Bill 291 notes 657 and 659, 292

Livingstone, David 72 n 114

Lockhart, Bill 292 n 671

Log(g)enberg family $368,369,370$ n 822, 374

Lombard, Hermanus Stephanus 536 n 1232 (H.J.), 592

Lombard, Ivanhoe Makepeace (Ivan) 300

Louw, Hoffie 422

Louw, Willem 263, 422

Lugg, Harry 473 n 1022, 554, 556

Lunguza ka Mpukane 282 n 633, 288

Lupini Brothers 456

Luthuli, F. 263

Magi (New Testament) 119

Mair, Alex 491, 492

Malan, David Eduard 360

Malan, François Stephanus 9, 27, 43 n 71, 141, 157, 168, 507,517

Malan, Jan 431

Malan, Johannes Augustinus 360

Malotho see Rampapoela, Piet

Mandy, John 298, 299

Manondu (Manondo,) 235, 236, 239, 243, 244, 247 , 254, 255

Manzi mDada 368, 371, 374, 377 n 841

Marais, Izak(?) 368, 370, 374

Maré, Estelle 457

Maré (Maree), Hester Maria Magdalena 321

Maré (Maree), Wynand Wilhelmus 321 n 754

Maritz, Gerhardus Marthinus (Gerrit, Gert) 91 n 162, $103,105,107,109,113-118,122,131,148,150$ n 294, 165, 221, 260 n 586, 283 n 639, 295, 319, 323 , $342,376,383,393,394,409,429,447$ n 956, 473 ,
476, 478, 482, 484 n 1086, 491, 498, 500, 501, 517-521

Maritz, Johannes Stephanus 226, 227, 481

Maritz, Susanna Catharina see Smit, Susanna

Mary (New Testament) 119

McKearin, Helen 291 n 663, 292

Meurant, Louis Henri 41 n 65, 113 n 196, 297, 299, 591 n 1354

Meyer, Coenraad Frederik 167, 185

Meyer, Debora Jacoba see Retief, Debora

Meyer (Mrs), Frikkie 511, 513

Meyer, Izaak Johannes 36

Meyer, Lucas Johannes 481

Meyer, Lucas Petrus Johannes (Piet) 165, 167, 182, 183 , 185

Minnaar, Karen 294 n 677

Minnaar, Maria Jacoba 148

Minnaar, Phil 595 n 1374, 597

Minnaar, Philippus Carel 148 n 281

Mitford-Barberton, Ivan Graham 39, 40

Mmemi ka Nguluzane 553 n 1270

Mncumbathe 89

Moaro see N[M?]wara

Möller, Lucy 449 n 977

Moerdyk (Moerdijk), Gerard Leendert Pieter 9, 13, 23, 27, 33, 36, 42, 47, 58, 61, 79, 90 n 149, 103, 137, $141,145,151-154,157,167,168,173,177,178,183$, $189,205,231,264,266,269,277$ n 612, 282, 286 $300,303,328-330,342,347,365,369,377,378$, $383,391,395,401,415,421,437,444$ n 952, 453, 456, 463, 465, 467-471, 495 n 1149, 502, 503 n $1175,507,513,517,519-521,525,539,541,550 \mathrm{n}$ $1260,556,558,561,566,577,581,594,596$

Moerdyk, Irma Leonora (married name Vermeulen) 177, 178, 503 n 1175

Moerdyk, Michel (Mike) 377, 378, 513

Moerdyk (née Pirow), Sylva Henriette 177, 178, 391

Montague, John 250, 394

Montgomery, John 90 n 156, 91 n 162, 131, 135, 148, 165 n 317, 221

Moodie, Duncan Campbell Francis 225 n 446, 279 notes 614 and 616,319 n 740, 488

Moolman, Jacobus Philippus 481

Morkel (Mr) 253

Moroka II (Moroko) 113, 139-154, 219 n 420

Moshweshwe 575, 590, 591, 595 n 1373

Moses (Old Testament) 116, 118, 122

Mostert, Dirk 36 n 41, 55 n 80, 98 n 181, 489 n 1106

Mpande kaSenzangakhona (Mpanda, Panda) $265 \mathrm{n}$ 600,439 n 946, 523-541, 553, 554, 557, 573

Mtshapi ka Noradu 553 n 1270

Muller, Christoffel 51 n 74, 83 n 129, 193 n 365, 216 n 404, 231, 232, 241 n 501, 242 n 503, 245 n 518, 246 n 524, 247, 260 n 586, 288 n 650, 369, 371, 372, 374, 485 n 1093

Mungo 281

Mzilikazi kaMashobane (Matselikatse, Musilicaats, Umselekaz) 81 n 127, 89, 91 n 160, 131, 135, 137, 147 n 275, 152, 165-167, 217, 218, 575 
Naidoo, Jay 231, 235 n 495, 250, 252, 260 n 586, 261, 264 n 593, 292 n 674

Napier, George Thomas 226, 233, 244, 475, 476, 573 , $574 \mathrm{n} 1303$

Napoleon 153, 451 n 986

Nathan, Manfred 72 n 114, 90 n 150, 98 n 178, 106, 107, 114 n 208, 167, 217 n 409, 249 n 533, 310, 311 n 733, 341, 342 n 785, 375 n 832, 377, 397, 409 n $873,426,429,449$ n 976, 518, 556 n 1282, 574 n 1305, 587, 589 n 1326, 590 n 1337, 593

Naudé, Philip Jacob 376

Ndlela kaSompisi (Umhlela) 225, 533

Ndukwana ka Mbengwana 282 n 633

Nduna ka Manqina 282, 553 n 1270

Nel, Philip Rudolph 502

Nel, Pieter 478 n 1045

Newton Thompson, Cyril 35, 36, 38, 41 notes 62 and 65 Newton Thompson, Joyce 35

Newton Thompson, William Rowland 35-39

Ngidi kaMcikaziswa 452, 553 n 1270, 554 n1272, 556 n 1278

Ngobese, Slindokuhle $291 \mathrm{n} 658$

N[M?]wara (Kwana, Moaro ) 235, 236, 239, 243, 244, $247,254,255$

Noble, John 488

Nongalaza kaNondela 536

Nonkalaza see Nongalaza kaNondela

Norden, Benjamin 165 n 313, 298

Oberholster, J.J. 29 n 24, 150 n 295, 223, 286 n 647, 319 n 739, 341 n 772, 518 n 1192, 521 n 1200, 589 notes 1327 and $1328,598 \mathrm{n} 1377$

Odé, G.A. 150, 358, 376 n 837

Oelofse, Catharina 391

O’Kulis, Dr see Postma, Willem

Oosthuizen (née Rudolph), Aletta Berhardina 360 n 810, 371 n 827, 376-377

Oosthuizen (Oosthuysen), Marthinus 235, 236, 239, 243, 247, 254, 255

Oosthuizen, Marthinus Jacobus 235 n 492, 303, 309, 360 n 810, 363-378

Opperman, P.H. 226

Otto, W. 426 n 898, 449-450 n 979

Owen, Charles Mostyn 590-592, 595

Owen, Francis 165, 167 n 329, 205, 209, 211, 215-223, 225, 231, 233, 235, 248-252, 260, 261, 264, 278-282, 319, 357 n 797

Pakington, John Somerset 593

Panda see Mpande

Parish (Cpt.) 591

Parker, Edward 228, 232, 233-234, 237, 240, 241, $245-246,447,534$

Parkington, John 288, 289 n 653

Pauw, Jack 73, 74

Pfeffer (Pfeiffer, Pheiffer), Johann Daniël Gottfried

(Daniel) 47, 51, 55, 58, 63, 67, 72-74

Philipps, Thomas 35, 38-39, 299

Phipson, Thomas 485, 487

Piers, C. 478, 480, 491
Pirow, Elza 513

Pirow (family), Oswald 513

Pirow, Sylva Henriette see Moerdyk, Sylva

Pistorius, Carl (brick layer) 476

Pistorius (Rev.), Carl Wilhelm Irene 473-474

Ploeger, Hendrik 109, 111, 196, 197, 211, 276

Postma, Laurika 19 n 18, 94, 98, 111, 119, 172-177, 303-305, 310-316 n 736, 326, 338, 347-353, 359, 360 n 811, 365-367, 373, 378, 383-391, 401-407, 415-422, 445, 471, 513, 515 n 1180, 545-550

Postma, Lenus 195, 391

Postma, Minnie 391

Postma, Philip 391

Postma, Stephanie 391

Postma, Sussie 195

Postma, Willem (pen name Dr O’Kulis) 119

Potgieter, Andries Hendrik 19, 54, 55, 69, 81, 89-99, 109, 113 n 199, 114-115, 127-136, 147-153, 165, 323, 357, 358, 376, 377, 393, 409, 430 n 916, 518, $575,587,589,591,593,594$

Potgieter (née Scholtz), Bettie 94

Potgieter, Carel 92, 93, 94, 132-136, 148 n 285, 149, 391

Potgieter, Catharina Helena 36

Potgieter, Ella see Stofberg, Ella

Potgieter, Evert Frederik (Bart) 232, 242-243, 245-248, 250, 254, 256-257, 260

Potgieter, Hennie $3,19,22,23,27,32,33,35,36$, 47-58, 61-67, 74, 79-87, 92, 93, 94, 95 n 176, 98, 103-111, 125-137, 149, 154, 157-163, 177, 191, 195, $263,265,315,325,341,359,377,389,395,407$, 421, 442, 452, 454, 469, 511, 515, 549, 563, 568, 587, 594 n 1368, 595

Potgieter, Hermanus Jacobus 149-150, 357-359, 447, 536 n 1232

Potgieter, Hermanus Philippus sr 149

Potgieter, Izak Meyer 36

Potgieter, Jacobus Christoffel (Koos Grootvoet) 409, 447 n 956,

Potgieter, Jacobus Johannes $143,145,149,150,152$, 154, 163 n 310, 447, 536 n 1232

Potgieter, Mathys Gerhardus Human 133

Preller, Gustav Schoeman 56, 57 n 89, 72 n 114, 148, 185, 217 n 409, 222, 231, 232, 233 n 474, 245 n 515, 251, 253 n 565, 256 n 572, 257, 260 n 586, 257, 260 n 586, 264-265, 298, 341-342 notes 779 and $781,376,378$ n 844, 397 n 864, 405 n 866, 411 n 877, 431, 448, 456, 457, 481 n 1060, 519 n 1198, 529 n 1204, 537, 539, 541

Preller (née Pretorius), Johanna Christina (G.S.) 185, 397 n 864

Pretorius, Andries Wilhelmus Jacobus 19, 23, 93-95, 89 n 179, 129, 185, 222-261, 285, 297, 395, 401-411, 415, 419, 426-432, 439-442, 447-457, 473, 475, 476, 481, 482, 484, 489, 501, 518, 522, 525-531, $536-541,573-577,582-595$

Pretorius, Christina Breggie 58

Pretorius, Debora Jacoba see Retief, Debora

Pretorius, Hannes 162

Pretorius, Henning Petrus Nicolaas 481, 484 
Pretorius Hercules Albertus (Bart) 232, 242, 243, 246, 247, 248, 250

Pretorius, Hilletje Anna Magdalena 341-342 n 779

Pretorius, Johanna Christina see Preller, Johanna

Pretorius, Johannes Petrus (Jan jr) 58, 73

Pretorius, Johannes Petrus (Jan sr) 592

Pretorius, Marthinus Wessel (1819-1901) 185 n 359, 225, 299, 585

Pretorius, Marthinus Wessel (Swart Martiens) 185 Pretorius, Quarta 95 n 177, 98 notes 179 and 183

Pretorius, Willem (Wilhelmus) Jurgen $87 \mathrm{n}$ 135, 90, 319 n 744, 357 n 800, 370, 371, 375, 376, 377, 449 n 977, 485 n 1094, 533 n 1215, 537 n 1237

Prinsloo, Magrieta 323

Prinsloo, Willem Petrus 199, 226

Proctor de Villiers see Kriel, Justa

Raath, Gerrit 451

Rampapoela, Piet 154

Ransford, Oliver 55, 90 n 150, 131 n 242, 450

Raper Peter E. 449 n 977

Raphael (Raffaello Sanzio da Urbino) 540

Rasmussen, Kent R. 89 n 137, 90 n 147, 191 n 159, 131, $136 \mathrm{n} 261$

Reitz, Francis William 299, 537, 538 n 1242

Retief, Debora Jacoba (married names Meyer, Landman, and Pretorius, see $181 \mathrm{n}$ 347) 165, 171-185, $503 \mathrm{n}$ 1175

Retief, Jacobus François 183

Retief, Gideon 185 n 358

Retief (née de Wet, widow of Jan Greyling), Magdalena Johanna (Lenie) 167, 182, 183, 185 n 358, 295, 473 n 1019

Retief, Magdalena Margaretha 182, 183

Retief, Pieter Cornelis 182, 183, 211, 220, 282, 397 n 864

Retief, Pieter Mauritz (Piet) 19, 42, 93, 98 n 179, 101-122, 129, 131, 150 n 294, 157, 165-170, 173, 175-185, 199-201, 203-266, 267-300, 305, 319, $323,326,330,341,342,357,362,376,395,397$ n $864,409,431,447,448,457,473,494,500,501$, $519,522,530,535,536,540,554$ n 1275, 556, 557, 573

Rheinallt-Jones, John David 329

Roberts(e), Fea 342, 343

Roberts(e), Heila Petronella 341, 342, 343

Roberts, Izaak Jacobus 341, 342

Roedolf (Rudolph), Bernhard 113

Roedolf, Coenie 22

Roedeloff (Rudolph), Johan Bern(h)ard 232, 248

Roedelof, P. 452

Roets, Hans 342 n 782

Romanelli, Romano (workshop) 4, 471, 549

Roodt-Coetzee, Kotie 513

Roos, François 534, 535

Roos, G.D. 162, 163 n 310

Roos, Janny 162,163 n 310

Roos, Jo 98 n 185, 500, 501

Rubens, Peter Paul 330, 332

Rudolph, Aletta Bernhardina see Oosthuizen, Aletta
Rudolph, Gerhardus Jacobus (Gert) 409

Rudolph, Jacobus Andreas sr 360 n 810, 376

Russell, John 593

Russell, Robert 488

Schalk, Willem Burger sr 360 n 809, 393, 395, 409

Samuel (Old Testament) 116, 118

Saul (Old Testament) 118

Scheepers family $342 \mathrm{n} 782$

Scheepers, Johannes Jacobus $58 \mathrm{n} 98$

Sche(e)pers, Marthinus Esaias $536 \mathrm{n} 1232$

Schmidt, A. 251

Scholtz, Bettie see Potgieter, Bettie

Scholtz, Joachim Hermanus 114

Scholtz, P.E. 592

Schuman, Theo 531

Schutte, P.(J.W.) 585, 592

Scott, Russell 3

Sekonyela (Sinka[n]jala, Sinko[n]yella, Sinkoyala), Mokotleng 166, 215-220, 234, 235, 236, 238, 243 , 244, 246, 247, 249, 254, 255, 259, 283

Shaka kaSenzangakhona 265 n 600 (Chaka), 283, 533

Sherson, A.N. 472, 475, 476, 477

Siebie (Stephan and Stephanie Joubert's dog) 178

Silberbauer, A. (Conrad Christian?) 298

Silevana 553, 556

Smit (née Botha), Anna 320

Smit, Christiaan Jacobus 147 n 274

Smit, Elizabetta Cecilia see Bezuidenhout, Elizabetta

Smit, Erasmus 43 n 70, 103-119, 147, 148, 150 n 294, $165-168,181,199,219,244-245,250,283,295$, 330, 341, 357 n 798, 393, 394, 397, 405, 409, 447 n 956, 473, 483

Smit, Nikolaas Christiaan 147, 148

Smit (née Maritz), Susanna Catharina 181, 295, 393-397, 574

Smit, Wynand 54

Smith (Maj.) $574 \mathrm{n} 1310$

Smith, Henry George Wakelyn (Harry) 55, 575, 576, 589, 590

Smith, Jesse and William 322-324

Smith, John Owen 73 n 115

Smuts, Jan Christiaan 31, 266, 328

Snijman (Snyman), C.F. 376

Snyman, Matthew $234 \mathrm{n} 486$

Sobhuza I (Sapusa) 556

Socwatsha ka Papu 553, 554

Sopoeza 545

Spanno, Lea 340, 341

Spies, Andries Theodorus $536 \mathrm{n} 1232$

Steenkamp, Alta 300 n 727

Steenkamp, Anna Elizabeth 519

Steenkamp, L.(S.?) 43 n 71, 79, 103, 141, 205, 269, 273 n $608,303,415,421,437,463,501,507,545,561$

Steyn, L.C. 325

Steynberg, Betsie $98 \mathrm{n} 183$

Steynberg, Coert Lourens 95, 98, 99, 419-422, $427-428,432-433,453,455,456,457,500,501$, 502

Steynberg, Isa 89, 96-99, 151 
Steytler, F.A. 157, 168, 189, 303, 330, 347, 365, 415, 437, 581

Stofberg (née Potgieter), Ella 92, 93, 94

Stofberg, Jan 94

Stoffberg (volksmoeder) 36

Stoffberg (Sen.) 36

Strydom, Hans 73 n 116

Strydom (née Terblanche), Martso 36, 94, 177

Stuart, James (1780-1853) 251

Stuart, James (1868-1942) 249, 280, 282 n 633, 288-289, 452, 553, 554 notes 1271,1272 and 1274 , 556 n 1278

Swart, Charles Robberts 39, 395

Tamburlaine (Amir Timur) 153

Tau the Lion 575

Tawana a Tlhutlwa 219 n 420

Terblanche, Martso see Strydom, Martso

Te Water, Charles Theodore 33

Theal, George McCall $83 n$ 129, 131, 132, 298, 431, 488, 575 n 1318, 590 n 1348, 592 n 1355, 593 n 1362

Theart-Peddle, Salomé 343, 395 n 860, 521 n 1201

Theunissen, N.H. 93 n 172, 132, 135, 136, 148 notes 278 and 285,149

Thom, Hendrik Bernardus 72 n 112, 115 n 213, 116, 318, 329, 474, 475, 478 n 1049, 481-485, 488, 489 notes 1111 and 1112, 490 notes $1114,1117,1119$ and 1121, 491 notes 1124,1125 and 1128, 516, 517 notes 1188 and 1190

Thompson, Leonard Monteath 58, 93 n 173, 119 n 223, 425-428, 430-433, 452, 473 n 1017, 475 n 1027, 482, 489, 590 n 1341

Thompson, William Rowland 31, 35-39, 297

Tikuba ka Magongo 553 n 1270

Trichardt, Anna 63, 65, 69

Trichardt, Carolus Johannes 72 n 114, 73, 545, 549

Trichardt, Louis 45-58, 59-75, 89, 98 n 179, 129, 154, $189,199,200,549$

Trichardt, Martha 49, 53-55, 57, 63-65, 69, 72, 73, 74

Trichardt, Petrus Frederik (Pieta) 73

Truter (Capt.) 422

Tununu ka Nonjiya 206-207, 209, 211, 248, 249, 280, $282 \mathrm{n} 633$

Tweed, Hugh 590 notes 1338 and 1343, 591 n 1351, 592-593 notes 1356 and 1357, 594 n 1369, 595 n 1373, 598 n 1377

Umhlela see Ndlela kaSompisi

Uys, Dirk Cornelis sr (Swart Dirk) $36 \mathrm{n} 42$

Uys, Dirk Cornelis (Dirkie) 36 n 42, 345-362, 377, 378, 447, 498, 515

Uys, Ian S. $135,357,360$

Uys, Jacobus Johannes 25-43, 119, 121, 297, 299

Uys, P. 537

Uys, Petrus Lafras (Piet) 35, 36 n 42, 131-135, 165, 347-362, 409, 430 n 916, 447

Vaandrager, Babette 94

Van Aswegen, J.A. 592

Van Bergen, Louis 35, 36, 37
Van den Berg, Zirk 13 n 5, 369 n 821

Van der Byl, Hendrik Johannes 594

Van der Linden (Linde), Johannes 13 n 4, 107, 115, 116, 118,585

Van der Lingen, G.W.A. 481

Van der Merwe, H. (Johannes?) 425

Van der Merwe, Johanna 323, 457

Van der Merwe, Pieter Jacobus 89-92, 95 n 175, 131, 135-137, 148 notes 281, 284 and 285, 151, 357 n 796, 457 n 1005

Van der Plank, Johan 482

Van der Walt, Andries $296 \mathrm{n} 684$

Van der Walt, Gert 94, 177 n 344, 341

Van der Walt (Dr) 422

Van Dyk, Elsie 94

Van Dyk, Joseph Sybrandt 342 n 782

Van Gass, Ferdinand Paulus 319 n 744

Van Heerden, Willem 407

Van Reenen (Mrs) 365, 394 n 857

Van Rensburg, B.H.J. 474

Van Rensburg, Hennie 109

Van Rensburg family 244 n 494, 303, 309, 365-378

Van Ren(s)burg, Johannis (Johannes, Hans) 369, 370 (n 822), 371, 376-378

Van Rensburg, P.J.J. 370, 375 n 832

Van Rijneveld, W.S. 425

Van Rooyen, G.H. 19 n 16, 159 n 308, 170, 175 n 341, 265 n 598, 295 n 681, 295 n 681, 297, 476 n 1036, 487 n 1096, 495

Van Schaik, J.L. 354, 515

Van Schalkwyk, François 234 n 486

Van Schoor, Tienie 531

Van Staden, Petrus Johannes(?) 226

Van Velden, Abraham 294-295

Van Velden, Dirk 294-295

Van Vooren, Petrus 342 n 782

Van Warmelo, Dietloff 389-391

Van Wouw, Anton 253, 495 n 1149

Van Zyl (student) 391

Venable, Henry Isaac 131 n 237, 225, 281, 323 n 760

Venter, Petrus Albertus 590-591

Verity, Thomas 216

Vermeulen, Irma Leonora see Moerdyk, Irma

Victoria (Queen) 216, 217, 574

Victory (goddess) 182

Viglione, Teresa 121, 316, 333-343

Viljoen, Benjamin Johannes (Ben) 299

Viljoen, Constand 457

Visagie, Jan C. 18, 19 n 14, 23 n 23, 34, 89 n 139, 112, 113 notes 198 and 199, 114 n 207, 147 n 274, 150 notes 292 and 294, 164, 165 n 312, 181 n 345, 185 n $358,198,297$ n 690, 341-342 notes 772 and 779 , $574 \mathrm{n} 1305$

Visagie (Johannes Hendrik?) 585

Waarzegger, Klaas see Meurant

Wagener, Gerrit 232, 242 n 503, 246 n 520, 253 n 566, 254, 258, 259

Walker, Eric Anderson 88, 114 n 203, 122, 362, 572

Warden, Henry Douglas 575, 589, 595, 597 
Weinthal, Leo 232, 246, 248, 250, 252-258, 264, 265

Willemse, Philip 13 n 5

Willemse, W.A. 325

William II 246

William IV 216

Wilson, Alexander 131 n 237

Wilson, Kenneth 292

Whimper, E. 286-287

Wolmarans, Frederik Gerhardus 589, 592
Wood, William 220 n 424, 221 n 426, 222, 248-249, 279-282, 294, 319

Yates, Alan 3, 109, 275, 286, 330

Zietsman, Johannes Philip 490, 529

Zietsman, Paul Hermanus 529, 536-537 (quote), 538, 556, 573

Zwartje (Marthinus Oosthuizen's horse) 368, 370, 374

Zuma, Jacob Gedleyihlekisa 459 